\title{
Management of osteoporosis in central and eastern Europe (CEE): conclusions of the "2nd Summit on Osteoporosis- CEE", 21-22 November 2008, Warsaw, Poland
}

\author{
Roman S. Lorenc • Heinrich Resch • \\ on behalf of the Members of the "2nd Summit on \\ Osteoporosis-Central and Eastern Europe (CEE)"
}

Received: 28 July 2009 / Accepted: 18 August 2009/Published online: 18 September 2009

(C) The Author(s) 2009. This article is published with open access at Springerlink.com

\begin{abstract}
In November 2008, the "2nd Summit on Osteoporosis - Central and Eastern Europe (CEE)" was held in Warsaw, Poland. Discussions at this meeting focused on the identification and discussion of diagnostic, preventive, and therapeutic measures used in CEE. Evaluated information was used to identify issues regarding diagnosis and therapy of osteoporosis in these countries to facilitate the subsequent setup of appropriate support and development strategies. The main debate was structured according to the following five subjects: (1) present status and future perspectives for implementation of FRAX $^{\circledR}$ into local (CEE) diagnostic algorithms, (2) principles of drug selection in osteoporosis treatment in CEE countries, (3) nonpharmacological interventions in osteoporosis treatment and prophylaxis in CEE countries, (4) treatment benefit evaluation, and (5) costeffectiveness and evaluation of reimbursement policies in CEE countries. The most important and substantial comments of the delegates are summarized in the present article. The multinational panel of experts with representatives from many CEE countries as well as Austria and Switzerland made the "2nd Summit on Osteoporosis - CEE" a perfect platform to identify issues and needs regarding diagnosis and therapy of
\end{abstract}

\section{R. S. Lorenc $(\square)$}

Department of Biochemistry \& Experimental Medicine,

The Children's Memorial Health Institute,

Al. Dzieci Polskich 20,

04-730 Warsaw, Poland

e-mail: biochemia@czd.pl

H. Resch

Medical Department (Rheumatology/Osteology

\& Gastroenterology), St. Vincent Hospital Vienna,

Medical University of Vienna,

Vienna, Austria osteoporosis as well as the cost-effectiveness of osteoporosis management in CEE countries. The information gained will serve as a basis for the development of strategies to resolve the identified issues at the "3rd Summit on Osteoporosis - CEE" in November 2009.

Keywords Central and eastern Europe $\cdot$ FRAX $^{\circledR} \cdot$ Diagnosis of osteoporosis · Treatment of osteoporosis $\cdot$ Health economics - Treatment benefit

\section{Introduction}

In Europe, USA, and Japan, about 75 million people suffer from osteoporosis [1]. During their lifetime, up to $50 \%$ of women and $30 \%$ of men will experience an osteoporosisrelated fracture [2]. Particularly in the case of hip fractures, immediate hospitalization is required that is quite often followed by a long and problematic recovery; in addition, a substantial number of patients become permanently disabled after such a fracture. This protracted course of the disease means that not only the patients' quality of life is considerably impaired but also that the costs for acute therapies and postoperative measures including rehabilitation are substantial. Hence, osteoporosis is one of the most serious chronic diseases that causes an enormous financial burden.

Because of their often serious consequences, prevention of fractures is the main goal of osteoporosis therapy. Prerequisite to achieve this goal is the identification of patients at risk for fractures by adequate diagnostic measures. Osteoporosis is characterized by low bone mineral density (BMD) [3]. Based on this parameter, guidelines for therapeutic interventions recommend assess- 
ing BMD for the diagnosis of osteoporosis $[4,5]$. The risk of fracture, however, is caused multifactorially, including risk factors such as age, prior fragility fractures, a parental history of hip fracture, smoking, use of systemic corticosteroids, excess alcohol intake, and rheumatoid arthritis [6, 7]. Therefore, BMD together with these factors should be considered when the fracture risk of an individual patient is evaluated $[6,7]$. Recently, the computer-based tool for fracture risk calculation (FRAX ${ }^{\circledR}$, http://www.shef.ac.uk/ FRAX/) has been developed. The algorithm of this tool takes this multifactorial approach into account. While rather simple to use, FRAX $^{\circledR}$ generates very reliable data on the individual fracture risk. Based on such information, physicians can then decide on the appropriate measures to be taken to prevent fractures.

In clinical practice, however, the diagnostic and therapeutic challenges of osteoporosis therapy are not always met. In fact, a substantial proportion of individuals at high risk, who have already had at least one fragility fracture, including hip fractures [8,9], are neither appropriately diagnosed nor treated for probable osteoporosis [10-12]. Simplifying the diagnostic procedure by such easy-to-use tools as FRAX ${ }^{\circledR}$ might increase the diagnosis rate of osteoporotic patients and support the timely administration of the required treatments. This tool, however, is not yet available in every country.

Therefore, the discussions at the "2nd Summit on Osteoporosis-Central and Eastern Europe (CEE)" concentrated on the need for appropriate discrimination and evaluation of the individual osteoporosis risk factors to maximize the benefits of pharmacotherapy while limiting the risks and costs that accompany treatment. Here, the major aim was to identify and discuss diagnostic, preventive, and therapeutic measures used in CEE. A comprehensive analysis covering these aspects in all CEE countries is not yet available, most probably because of considerable differences between the individual countries not only regarding culture, living conditions, life expectancy but also regarding availability and use of medical treatment for osteoporosis and, finally, reimbursement.

Representatives from Austria and Switzerland but mainly from CEE countries including the Czech Republic, Hungary, Poland, Romania, Slovakia, and Slovenia participated in the "2nd summit on Osteoporosis-CEE". This multinational panel of experts made the meeting a perfect platform to develop the above topics. Discussion was based on six international reference publications [13-18]; the main debate was structured according to the following five subjects:

1. Present status and future perspectives for implementation of FRAX ${ }^{\circledR}$ into local (CEE) diagnostic algorithms

2. Principles of drug selection in osteoporosis treatment in CEE countries
3. Nonpharmacological interventions in osteoporosis treatment and prophylaxis in CEE countries

4. Treatment benefit evaluation

5. Cost-effectiveness and evaluation of reimbursement policies in CEE countries

The information evaluated during the summit was used to identify issues regarding diagnosis and therapy of osteoporosis in CEE countries to facilitate the subsequent setup of appropriate support and development strategies. The most important and substantial comments of the delegates are summarized below.

\section{Present status and future perspectives for implementation of FRAX® into local (CEE) diagnostic algorithms}

The computer-based tool FRAX ${ }^{\circledR}$ has been developed by the WHO from studying population-based cohorts from Europe, North America, Asia, and Australia to evaluate the fracture risk of patients (http://www.shef.ac.uk/FRAX/). It is based on individual patient models that integrate the risks associated with clinical risk factors as well as BMD at the femoral neck. FRAX ${ }^{\circledR}$ represents a very sensitive tool to identify patients with a high fracture risk; its output is a 10year probability of hip fracture and the 10-year probability of a major osteoporotic fracture (clinical spine, forearm, hip, or shoulder fracture). Use of FRAX ${ }^{\circledR}$ for fracture risk calculation is recommended by current European guidelines [15] as well as by guidelines of the National Osteoporosis Foundation and the World Health Organization (WHO) [19] to improve diagnosis, facilitate the decision for appropriate therapeutic interventions, and, in the long run, save costs by fracture prevention.

Prerequisite for the implementation of FRAX $^{\circledR}$ in a specific country is information on the local epidemiology of fractures and death. In several countries including UK, Germany, Sweden, Japan, the USA, and others, sufficient reliable epidemiological data are available to calculate the fracture risk of an individual patient by FRAX $^{\circledR}$. In countries where such epidemiological data are missing, the guidelines for the use of FRAX $^{\circledR}$ recommend to "use (the FRAX ${ }^{\circledR}$ model of) the country for which the epidemiology of osteoporosis most closely approximates your country." (http://www. shef.ac.uk/FRAX/). However, this approach is in a way problematic because the incidence of hip fractures and death differ considerably (10- to 15-fold) between countries [20-22]. Hence, to obtain reliable information on fracture risk by FRAX ${ }^{\circledR}$, local data on fracture and death rates should be assessed before implementation of FRAX $^{\circledR}$ in a specific country. 
Comments of the delegates on the use of FRAX ${ }^{\circledR}$ in CEE countries

Expectations from FRAX® in CEE countries The final aim of any interventional procedure in osteoporosis is the development of a uniform, diagnostic, therapeutic, and cost-effective algorithm of treatment and fracture prevention.

FRAX $^{\circledR}$ has been developed as a 10 -year fracture risk calculator based on femoral neck densitometry (or BMD) and available independent clinical fracture risk factors. FRAX $^{\circledR}$ generally does not change WHO diagnostic classification of osteoporosis; however, with its use, we can expect that a lower number of younger patients at low risk as well as a higher number of elderly patients at high risk will be selected for treatment.

FRAX $^{\circledR}$ can be a helpful screening tool in general practitioner case-finding strategies to identify patients with a high fracture risk (diagnostic threshold) without the use of densitometry or with only limited access to densitometry.

Specific issues regarding the use of FRAX® in CEE countries The audience agreed that presently, there are insufficient, satisfactorily validated studies concerning spine densitometry and fracture rates available in CEE that can be utilized in the FRAX ${ }^{\circledR}$ algorithm.

Prior to the implementation of FRAX $^{\circledR}$ into national routine guidelines, reliable local fracture data need to be assessed for each CEE country to enable appropriate risk assessment and local cost-effectiveness calculation of the whole procedure.

As a next step, the implementation of FRAX ${ }^{\circledR}$ software in routine densitometry devices could be helpful in everyday diagnostic procedures in CEE countries. Through this, a consistent and reliable diagnosis of osteoporosis could be guaranteed cross-nationally in CEE. Besides, the effort for physicians would be minor because of the straightforward methodical approach of FRAX ${ }^{\circledR}$.

The following practical remarks and questions were raised by the summit participants Is it possible that the history of nontraumatic osteoporotic fractures as well as low lumbar (beside hip) BMD values ( $T$ score of $\leq 2.5$ standard deviation) could define a more precise diagnostic threshold with the aim of improving an interventional threshold for pharmacological treatment? It was agreed that including such fracture information will not yield better results.

In summary, all representatives of the CEE countries expressed interest in the use of FRAX ${ }^{\circledR}$ for fracture risk evaluation in osteoporotic patients. The discussion, however, made clear that currently, the FRAX ${ }^{\circledR}$ model cannot be implemented in CEE countries because reliable fracture data are not available to adjust the model to the special circumstances in each individual CEE country. Since the beginning of 2009, a FRAX ${ }^{\circledR}$ model based on Austrian fracture data is available. The authors suggest that in accordance with http://www.shef.ac.uk/FRAX, Austria might be used as a surrogate country for CEE countries until sufficient data for the establishment of a CEE-specific FRAX $^{\circledR}$ algorithm are available.

\section{Principles of drug selection in osteoporosis treatment in $\mathrm{CEE}$ countries}

A range of drugs is available for the therapy of osteoporosis that significantly reduces the risk of vertebral and nonvertebral fractures $[15,19]$. Most commonly used drugs are selective estrogen receptor modulators such as raloxifene; bisphosphonates such as alendronate, risedronate, ibandronate, and zoledronate; parathyroid hormone (PTH)-derived drugs such as teriparatide; and so-called dual action bone agents (DABA) including strontium ranelate. In addition, hormone replacement therapies (females, estrogen; males, testosterone) can be used $[15,19]$.

The decision for one or more of these therapeutic approaches is based primarily on the fracture risk of an individual patient $[4,5]$ but also on biochemical markers for bone turnover $[6,7]$. In addition, individual patient's characteristics should be considered: Will the patients comply with a therapy? Are they able to swallow their medication or do they need intravenous application? How is their individual tolerability to a certain drug? etc.

Comments of the delegates on drug selection in CEE countries

Treatment decisions in osteoporosis should be based on a multifactorial approach Treatment decisions in osteoporosis should be based on the absolute risk of fracture (when possible by use of FRAX ${ }^{\circledR}$ ) which combines the patient's clinical risk factors with BMD values. In all cases of low bone mass or low-trauma fractures, metabolic disorders as secondary causes of osteoporosis should be ruled out; however, causative management of secondary osteoporosis does not exclude the need for antifracture pharmacotherapy. In selected patients, an assessment of bone turnover rates using biochemical markers of bone turnover could possibly influence the selection of the most appropriate treatment.

General considerations on drug selection Drug selection in osteoporosis treatment should take into account the mechanism of action of the drug and the results of randomized, placebo-controlled clinical trials demonstrating the effects of a given intervention on fracture risk. Comorbidities as well as nonskeletal risks and benefits of the drug should also be considered. 
Osteoporosis is a chronic disease. Therefore, long-term adherence (compliance and persistence) to the treatment is as important as effectiveness. The suitability of the drug for longterm administration and factors such as patient's preference, tolerability, and convenience should be taken into account.

Anticatabolic drugs are most appropriate in patients with high bone turnover, while anabolic drugs demonstrate efficacy irrespective of bone turnover. Anabolic treatment should be chosen particularly in patients with low bone formation or extremely low bone mass, in elderly, in cases of glucocorticoid-induced osteoporosis, or after multiple fractures where preservation of bone mass and bone architecture by antiresorptive drugs is not sufficient to reduce the high absolute risk of fracture efficiently.

Recent studies give evidence that sequential treatment with anabolic followed by anticatabolic drugs may preserve and even improve the gain in bone mass needed for longterm efficacy.

Bisphosphonates All bisphosphonates are highly effective in postmenopausal female and male patients with established osteoporosis, especially in those with high bone turnover. Presently, there is no evidence of any effect of bisphosphonates in osteopenia.

The main differences among various oral bisphosphonates relate mostly to compliance and persistence (adherence to therapy). A once-a-month schedule is better accepted by patients than once a week, which, in turn, seems to be better than a daily schedule.

Intravenous bisphosphonates (ibandronate, $3 \mathrm{mg}$ every 3 months; zoledronate, $5 \mathrm{mg}$ once a year) may be particularly useful in the treatment of patients with gastrointestinal disorders and patients intolerant to oral bisphosphonates, as well as patients who are chronically immobilized (as a result of vertebral or hip fractures; stroke patients), or with dementia. Once yearly zoledronic acid therapy does not only maintain bone microarchitecture but also enables sufficient bone preservation. Moreover, apart from the bone-preserving effect, zoledronic acid administered once a year guarantees high adherence to therapy.

Dual action bone agents Strontium ranelate, with its synchronous antiresorptive and pro-anabolic effects, shows antifracture efficacy in all types of osteoporotic fractures, both vertebral and nonvertebral, regardless of initial BMD or bone turnover. Moreover, a statistically significant reduction in the incidence of femoral fractures in older women with low bone mineral density can be shown.

Parathyroid hormone-derived drugs Teriparatide is a highly effective bone anabolic agent; treatment studies show a highly significant reduction of osteoporotic fractures of any type in patients with severe osteoporosis. Presently, it is the only medication which restores bone structure independently of the degree of initial disarrangement. For safety reasons, however, the duration of treatment has been restricted to 24 months. In order to maintain the achieved therapeutic effects, continuation of treatment with bisphosphonates should be considered.

In summary, drug selection should be based not only on physical (absolute risk of fractures, biochemical markers of bone turnover, etc.) but also on patient-specific (comorbidities, patient's preference, tolerability, ability to comply, etc.) factors. In addition, the mode of action of a drug should match the pathological characteristics (e.g., high/low bone turnover) of an individual patient. Specifically, anticatabolic drugs are most appropriate in patients with high bone turnover, while anabolic drugs demonstrate efficacy irrespective of bone turnover. To achieve longterm efficacy, sequential treatment with anabolic followed by anticatabolic drugs should be considered. All bisphosphonates are highly effective in postmenopausal patients with established osteoporosis; decision on oral vs. intravenous formulations as well as on the application schedule should depend on patient characteristics. DABAs are suitable for all types of fractures irrespective of BMD and bone turnover. The PTH-derived drug teriparatide is currently the only formulation which restores bone structure also in patients suffering from severe osteoporosis.

\section{Nonpharmacological interventions in osteoporosis treatment and prophylaxis in CEE countries}

Besides proper medication, a multitude of further measures have been demonstrated to reduce the risk of osteoporosis, osteoporosis-related fractures, and fallrelated injuries. Physical activity, for example, has not only a positive impact on bone mineral density [23-25] but also prevents falls especially in elderly patients by increasing their balance and physical confidence [26]. Adequate intake of calcium supports the positive effect of physical activity [27] and increases bone mineral density [28]. Regular intake of vitamin D reduces the risk of falls and the fracture risk $[29,30]$.

Comments of the delegates on nonpharmacological interventions and prophylaxis in CEE countries

Considerations regarding vitamin D supplementation During any osteoporosis therapy, vitamin D status should be optimized (serum $25 \mathrm{OHD}>30 \mathrm{ng} / \mathrm{ml}$ in serum) for a proper antifracture effect.

Preventions of falls and hip fracture risk reduction are evidenced for vitamin D supplementation in vitamin D- 
deficient patients. The recommended daily dose of vitamin D should range between 800-2,000 IU.

Patients with decreased renal function should be supplemented with activated vitamin D metabolites.

A history of kidney stones or hypercalciuria needs further evaluation before initiating vitamin D supplementation.

Other considerations Many nonspinal fractures result from a fall. Hence, each elderly patient should be asked about falls; if one or more are reported, a multidisciplinary program should be implemented.

General practitioners should provide printed educational materials with information on prophylaxis such as fall prevention, proper daily exercise, adequate lifestyle changes, etc.

Spinal dysfunction and peripheral joint pain limiting movement as well as weakening of muscles should be considered as indications for rehabilitation.

Besides vitamin D intake, calcium supplementation is the main approach used in fracture prevention and the necessary complement to osteoporosis treatment. The recommended daily dose of calcium is 500-1,500 mg.

In summary, besides calcium and vitamin D supplementation, the panel members underlined the importance of comprehensively enquiring about the medical history of patients, proper education as well as the use of multidisciplinary approaches in the prevention and treatment of osteoporosis.

\section{Treatment benefit evaluation in CEE countries}

Monitoring the effects of osteoporosis therapy informs the physician whether a certain treatment was efficient or not. Besides fracture rates, several surrogate markers are employed to evaluate the outcome of osteoporosis therapy. The most commonly used surrogate markers are sequential measurements of BMD and bone turnover markers (BTMs). Stable or increasing BMD and suppressed BTMs are associated with a reduction in fracture risk [31-34]. The available surrogate measurements of bone strength which are applied to assess the effects of osteoporosis treatment were intensively discussed by the participants.

Comments of the delegates on treatment benefit evaluation

General considerations Presently, BMD measurements are the most widely used and probably the best long-term assessment of the efficacy of antifracture treatment. Bone turnover can be monitored using BTMs (CTX, P1NP, OC). However, the usefulness of these markers in the clinical practice as a short-term (at 3 months) surrogate monitoring tool in patients treated with antiresorptives (bisphosphonates, raloxifene, hormone therapy, calcitonin) or anabolic (PTH) drugs needs to be further validated. In both BTMs and BMD measurements, precision standards and quality control by calculation of the least significant change (LSC) for the biochemical assays and BMD measurements should be taken into account for the interpretation of the individual results. Only compliant patients may be defined as "nonresponder" or "suboptimal responder" when no significant changes (according to LSC) of BMD or BTMs are observed during treatment. A patient is defined as compliant when she/he correctly takes at least $80 \%$ of the prescribed doses of the treatment in a minimal time interval of 1 year.

The most controversial point appeared to be the question whether an incident fracture is a reliable clinical endpoint for evaluation of a therapy's effectiveness. Fractures do not appear as uniform events being very heterogeneous depending on the analyzed country. On the one hand, a fracture is a stochastic event (i.e., subject to randomness) that may or may not occur in an osteoporotic patient regardless of the treatment. On the other hand, fracture prevention is the primary aim of osteoporosis treatment. However, the incident fracture rate has been defined as the primary endpoint in all relevant osteoporotic clinical trials but with potential limits when judged in a single patient. It was agreed that an incident fracture is not necessarily a treatment failure. However, if the fracture occurs a considerable time after the commencement of the treatment, the need for drug change (anticatabolic to anabolic) and specific nonpharmacological intervention (fall prevention, balance training, muscle strengthening) should be considered, if possible.

The general conclusion of all participants led to the statement that treatment benefit evaluation can be considered one of the most important factors to improve long-term antifracture efficacy. Since there are no direct tools for bone strength measurement in living patients, we are presently limited to existent surrogate ones.

\section{Cost-effectiveness and evaluation of reimbursement policies in CEE countries}

As a chronic disease that in many cases is accompanied by limiting complications and consequences, osteoporosis is a very treatment- and, hence, cost-intensive condition. Considering also the high prevalence of this disease, the burden imposed by osteoporosis on health care systems is enormous. Especially in countries with limited resources and health care budgets, the decision taken on diagnostic and therapeutic measures to prevent or treat osteoporosis has to be based not only on therapeutic but also on economic considerations. Health-economy analyses that 
evaluate the cost/benefit ratio of a certain therapy can support the efficient allocation of such limited resources. Indeed, economic evaluations have been performed to compare distinct treatment strategies in osteoporosis [3537]. However, to realistically represent the cost/benefit of these therapies in a certain area or country, detailed local data on epidemiology, type of treatment, treatment expenses, success rates, etc. have to be incorporated into the evaluation.

Difficult and heterogeneous economic situation in CEE Due to historical developments, the economic situation in CEE countries shows large differences also regarding budgets available for health care. Besides countries with sufficient financial means for health care, there are also a considerable number of countries where resources are limited. Costeffectiveness analyses provide important information about the value of different treatment options. Their outcome assists decision makers who try to equitably allocate constrained resources in order to achieve maximum health care benefits. By definition, cost-effectiveness analyses compare the costs and health effects of an intervention to assess whether it is worth doing from an economical perspective. From an ethical point of view, economically dealing with resources is a must.

Data required for cost-effectiveness analyses are not available With respect to osteoporosis and costeffectiveness, reliable epidemiological data and the exact cost of osteoporosis and fracture treatments are necessary as well as data on normal life expectancy and gross national product. While in Poland, the costeffectiveness of alendronate and raloxifene (once a day) as well as ibandronate (once a month) therapies for postmenopausal osteoporosis were evidenced and published [38], in most other CEE countries, the cost of osteoporosis treatment is not available. One of the reasons that should be considered in this context is that registers for hip fractures and other fractures are far from being satisfactory in this region.

Reimbursement strategies are very heterogeneous in distinct CEE countries Only in some CEE countries do uniform reimbursement criteria exist. In general, however, reimbursement of diagnostic procedures, prevention, and therapy of osteoporosis varies considerably among countries as well as factors influencing reimbursement policies. In fact, there are currently no recommendations on how and to what extent reimbursement policies in CEE countries should be influenced by cost-effectiveness analyses. In addition, in the majority of CEE countries, medical communities are not involved in cost-effectiveness evaluation.
Which substances are reimbursed in CEE In the majority $(>50 \%)$ of CEE countries, alendronate, risedronate, ibandronate, zoledronic acid, strontium ranelate, and teriparatide/ rh PTH are reimbursed; however, while in some countries, therapy costs are fully covered, in other countries, costs are reimbursed only partially. Raloxifene is not reimbursed at all in some countries.

Recommendations for the future In the long run, the main approach should focus on (a) the development of CEEspecific FRAX ${ }^{\circledR}$ algorithms to guarantee reliable diagnosis, thereby increasing the efficacy of therapeutic measures, and (b) country-specific cost-effectiveness models to facilitate calculation of regional therapy costs. Such models of the cost-effectiveness of antifracture therapies would enable the assessment and comparison of different drugs (alendronate, ibandronate, risedronate, raloxifene, strontium ranelate, zoledronic acid), different screening strategies (BMD, BTM, DXA), or patients of different ages and sex.

In summary, the CEE delegates pointed out the heterogeneous economic situations in CEE countries and emphasized that country-specific health-economy analyses are required to shed light on the cost-effectiveness of local osteoporosis therapies. However, the data required to conduct such analyses (epidemiology data, exact costs of therapies, etc.) are not available. In addition, reimbursement strategies vary considerably between countries, making an objective evaluation of local situations even more difficult. As a future perspective, they suggested that diagnosis and therapy of osteoporosis should be based on the concerted use of CEE-specific FRAX ${ }^{\circledR}$ algorithms and local costeffectiveness data.

\section{Overall summary and outlook}

The major aim of the "2nd summit on Osteoporosis - CEE" was to identify and discuss diagnostic, prophylactic, and therapeutic measures used in CEE countries to prevent and treat osteoporosis [39]. Based on such information, issues concerning the management of osteoporosis in these countries should then be identified to provide the basis for the development of suitable support and development strategies.

It was agreed that a proper diagnosis especially of the patient's fracture risk is the absolute prerequisite for the decision on an adequate and successful therapy. To facilitate a simple but reliable diagnose of the fracture risk, all representatives of the CEE countries argued for the implementation of the computer-based tool FRAX ${ }^{\circledR}$ for fracture risk evaluation in CEE. For this, information on the local epidemiology of fracture and death rates is required. 
Such data, however, are currently not available for most countries in CEE. Therefore, the first step toward FRAX ${ }^{\circledR}$ implementation would be to develop a strategy on how such information can be collected most efficiently in CEE countries. Until sufficient local epidemiology data for the establishment of CEE-specific FRAX $^{\circledR}$ algorithms are available, the Austrian FRAX ${ }^{\circledR}$ model was proposed as a surrogate model for CEE.

Drug selection should be based on physical parameters (absolute risk of fractures, biochemical markers of bone turnover, etc.) as well as on patient-specific factors (comorbidities, patient's preference, tolerability, ability to comply, etc.). Such patient characteristics should also be considered when deciding on the way of administration (oral vs. intravenous) and the administration schedule (daily up to once a year). The most commonly used therapies are bisphosphonates, DABA, and PTH-derived formulations. In addition to such medication-based treatments, calcium and vitamin D supplementation were discussed to be vital for a successful therapy as well as measures such as comprehensively enquiring about the patient's medical history, proper education, and, in general, the use of multidisciplinary therapeutic approaches. For evaluation of the benefit of a specific osteoporosis treatment, no direct tools are currently available; therefore, surrogate ones have to be employed.

Finally, the CEE delegates described the very heterogeneous economic situations in the different CEE countries and emphasized that country-specific health-economy analyses would be required to shed light on the costeffectiveness of local osteoporosis therapies. For such studies, however, epidemiology data, data on the exact costs of therapies, etc. have first to be evaluated in each country. As soon as available, the combined use of CEEspecific FRAX ${ }^{\circledR}$ algorithms and local cost-effectiveness data would then allow an adequate and economical management of osteoporosis.

In conclusion, the lively discussion and exchange of information at the "2nd Summit on Osteoporosis - CEE" in November 2008 made clear that this meeting is a very helpful and authentic platform to identify issues and needs regarding the diagnosis and therapy of osteoporosis as well as the cost-effectiveness of osteoporosis management in CEE countries. Based on the information gained, the "3rd Summit on Osteoporosis-CEE" in November 2009 will then focus on the development of answers and strategies to resolve the identified issues.

Acknowledgment The organization of the CEE Summit was supported by an unrestricted educational grant of AMGEN.

CEE Summit Members H. Dimai, G. Holzer, Austria; P. Broulik, P. Horák, J. Rosa, J. Stepan, Czech Republic; C. Horvath, P. Lakatos, I. Marton, L. Szekeres, Z. Valkusz, Hungary; E. Czerwiński, E. Franek,
P. Głuszko, W. Horst-Sikorska, E. Karczmarewicz, K. KsiężopolskaOrłowska, P. Leszczyński, W. Misiorowski, J. Przedlacki, A. Więcek, Poland; D. Opris, C. Poiana, Romania; Z. Killinger, P. Masaryk, J. Payer, Slovakia; J. Preželj, Slovenia; D. Hans, Switzerland.

Open Access This article is distributed under the terms of the Creative Commons Attribution Noncommercial License which permits any noncommercial use, distribution, and reproduction in any medium, provided the original author(s) and source are credited.

\section{References}

1. EFFO and NOF (1997) Who are candidates for prevention and treatment for osteoporosis? Osteoporos Int 7:1-6

2. Randell A, Sambrook PN, Nguyen TV et al (1995) Direct clinical and welfare costs of osteoporotic fractures in elderly men and women. Osteoporos Int 5:427-432

3. Consensus Development Conference (1993) Diagnosis, prophylaxis and treatment of osteoporosis. Am J Med 94:646-650

4. Kanis JA, Johnell O (2005) Requirements for DXA for the management of osteoporosis in Europe. Osteoporos Int 16:220-238

5. Blake GM, Fogelman I (2007) Role of dual-energy X-ray absorptiometry in the diagnosis and treatment of osteoporosis. J Clin Densitom 10:102-110

6. Kanis JA, Borgstrom F, De Laet C et al (2005) Assessment of fracture risk. Osteoporos Int 16:581-589

7. Kanis JA, Oden A, Johnell O et al (2007) The use of clinical risk factors enhances the performance of BMD in the prediction of hip and osteoporotic fractures in men and women. Osteoporos Int 18:103-1046

8. Rabenda V, Mertens R, Fabri V et al (2008) Adherence to bisphosphonates therapy and hip fracture risk in osteoporotic women. Osteoporos Int 19:811-818

9. Rabenda V, Vanoverloop J, Fabri V et al (2008) Low incidence of anti-osteoporosis treatment after hip fracture. J Bone Joint Surg Am 90:2142-2148

10. Freedman KB, Kaplan FS, Bilker WB et al (2000) Treatment of osteoporosis: are physicians missing an opportunity? J Bone Joint Surg Am 82-A:1063-1070

11. Siris ES, Miller PD, Barrett-Connor E et al (2001) Identification and fracture outcomes of undiagnosed low bone mineral density in postmenopausal women: results from the National Osteoporosis Risk Assessment. JAMA 286:2815-2822

12. Nguyen TV, Center JR, Eisman JA (2004) Osteoporosis: underrated, underdiagnosed and undertreated. Med J Aust 180:S18-S22

13. Kanis JA, McCloskey EV, Johansson $\mathrm{H}$ et al (2008) Case finding for the management of osteoporosis with FRAX $^{\circledR}$ — assessment and intervention thresholds for the UK. Osteoporosis Int 19:1395-1408

14. Kanis JA on behalf of the World Health Organization Scientific Group (2008) Assessment of osteoporosis at the primary health care level. Technical report. World Health Organization Collaborating Centre for Metabolic Bone Diseases. University of Sheffield, UK. Summary report. Fracture Risk Assessment Tool (FRAX ${ }^{\mathrm{TM}}$ )

15. Kanis JA, Burlet N, Cooper C et al (2009) European guidance for the diagnosis and management of osteoporosis in postmenopausal women. Osteoporos Int 19:388-428

16. Zethraeus N, Borgström F, Ström O et al (2007) Cost-effectiveness of the treatment and prevention of osteoporosis - a review of the literature and a reference model. Osteoporos Int 18:9-23

17. Lorenc R.S, Głuszko P, Karczmarewicz E et al (2007) Recommendation on the diagnosis and treatment of osteoporosis in Poland. Reducing the incidence of fractures through effective 
prevention and treatment. http://www.iofbonehealth.org. Accessed Jun 2009

18. Dimai HP, Pietschmann P, Resch H et al (2002) Leitfaden zur medikamentösen Therapie der postmenopausalen Osteoporose. Wien Med Wschr 152:596-612

19. Lewiecki WNB, EM MPD et al (2008) National Osteoporosis Foundation 2008 Clinician's Guide to Prevention and Treatment of Osteoporosis and the World Health Organization Fracture Risk Assessment Tool (FRAX): what they mean to the bone densitometrist and bone technologist. J Clin Densitom 11:473477

20. Elffors I, Allander E, Kanis JA et al (1994) The variable incidence of hip fracture in southern Europe: the MEDOS Study. Osteoporos Int 4:253-263

21. Johnell O, Gullberg B, Allander E et al (1992) The apparent incidence of hip fracture in Europe: a study of national register sources. Osteoporos Int 2:298-302

22. Kanis JA, Johnell O, De Laet C et al (2002) International variations in hip fracture probabilities: implications for risk assessment. J Bone Miner Res 17:1239-1244

23. Kemmler W, Lauber D, Weineck J et al (2004) Benefits of 2 years of intense exercise on bone density, physical fitness, and blood lipids in early postmenopausal osteopenic women: results of the Erlangen Fitness Osteoporosis Prevention Study (EFOPS). Arch Intern Med 164:1084-1091

24. Preisinger E, Alacamlioglu Y, Pils K et al (1995) Therapeutic exercise in the prevention of bone loss. A controlled trial with women after menopause. Am J Phys Med Rehabil 74:120-123

25. Hartard M, Haber P, Ilieva D et al (1996) Systematic strength training as a model of therapeutic intervention. A controlled trial in postmenopausal women with osteopenia. Am J Phys Med Rehabil 75:21-28

26. Province MA, Hadley EC, Hornbrook MC et al (1995) The effects of exercise on falls in elderly patients. A preplanned meta-analysis of the FICSIT Trials. JAMA 273:1341-1347

27. Specker B, Binkley T (2003) Randomized trial of physical activity and calcium supplementation on bone mineral content in 3- to 5year-old children. J Bone Miner Res 18:885-892
28. Shea B, Wells G, Cranney A et al (2002) Meta-analyses of therapies for postmenopausal osteoporosis. VII. Meta-analysis of calcium supplementation for the prevention of postmenopausal osteoporosis. Endocr Rev 23:552-559

29. Bischoff-Ferrari HA, Willett WC, Wong JB et al (2005) Fracture prevention with vitamin $\mathrm{D}$ supplementation: a meta-analysis of randomized controlled trials. JAMA 293:2257-2264

30. Bischoff-Ferrari HA, Dawson-Hughes B, Willett WC et al (2004) Effect of vitamin D on falls: a meta-analysis. JAMA 291:1999-2006

31. Lewiecki EM, Watts NB (2008) Assessing response to osteoporosis therapy. Osteoporos Int 19:1363-1368

32. Reginster JY, Collette J, Neuprez A et al (2008) Role of biochemical markers of bone turnover as prognostic indicator of successful osteoporosis therapy. Bone 42:832-836

33. Bouxsein ML, Delmas PD (2008) Considerations for development of surrogate endpoints for antifracture efficacy of new treatments in osteoporosis: a perspective. J Bone Miner Res 23:1155-1167

34. Bonnick SL, Shulman L (2006) Monitoring osteoporosis therapy: bone mineral density, bone turnover markers, or both? Am J Med 119(4 Suppl 1):S25-S31

35. Strom O, Borgstrom F, Sen SS, Boonen S et al (2007) Costeffectiveness of alendronate in the treatment of postmenopausal women in 9 European countries - an economic evaluation based on the fracture intervention trial. Osteoporos Int 18:1047-1061

36. Jonsson B, Christiansen C, Johnell O, Hedbrandt J et al (1996) Cost-effectiveness of fracture prevention in established osteoporosis. Scand J Rheumatol Suppl 103:30-38

37. Stevenson M, Lloyd Jones M, De Nigris E et al (2005) A systematic review and economic evaluation of alendronate, etidronate, risedronate, raloxifene and teriparatide for the prevention and treatment of postmenopausal osteoporosis. Health Technol Assess 9:1-160

38. Karczmarewicz E, Szkultecka-Debek M, Lorenc RS (2007) Pharmacoeconomical analysis in osteoporosis. Pharmacoeconomics 11:3-9

39. Franek E, Karczmarewicz E, Misiorowski W et al (2009) Treatment benefits evaluation in osteoporosis - position of the summit international conference on osteoporosis - central eastern Europe held 2122 November 2008 Warsaw, Poland. Bone 44:S374-S375 